comparability of experimental conditions and subjects, the fact that such discrepancies can exist in the only reasonably well-documented part of the subject illustrates how little has yet been done.

The great differences in response to climatic stress between a sweating and a nearly non-sweating mammal were made very plain by J. D. Findlay (Hannah). In a dry atmosphere, increase in environmental temperature well above normal deep-body temperature in man can be tolerated by great increases in rate of sweating and elimination of heat by evaporation (the classical experiment proved survival at $127^{\circ}$ C.). The cow has a type of poorly vascularized sweat gland, but approaches its sweating maximum at about $26^{\circ} \mathrm{C}$., above which loss of heat by panting is substituted. Panting in turn reaches its maximum effectiveness at about $40^{\circ} \mathrm{C}$., which is an almost lethal temperature.

Information on other aspects of interaction of climatic stress and nutrition is sparse. Discussion by R. H. Fox (Medical Research Council Laboratories) and by Blaxter and others on the changes in requirement for vitamins, and on losses of amino-acids and minerals through the skin, and the relation of these to such deficiency-diseases as pellagra and kwashiorkor were speculative.
S. D. Morrison

\title{
X-RAY RESEARCH
}

$\mathrm{T}$ HE Spring Conference of the X-ray Analysis Group of the Institute of Physics was held in the College of Science and Technology, Manchester, on April 18 and 19. No particular subject was fixed for discussion, and papers were invited on any topic in the field of crystallography and X-ray analysis.

A novel feature of the Conference was a partsession devoted to the reading of five papers by research students. Although there have, of course, been papers at previous conferences of which research students have been authors or joint authors, the Group committee felt that to set aside a period for such papers might particularly encourage research students to feel that their work was of value and worthy of the attention of the Group as a whole. The five papers discussed were selected from some ten papers offered.

Mr. J. V. P. Long (Cavendish Laboratory, Cambridge) described experiments on the use of fine-focus $X$-ray tubes and X-ray absorption and emission spectra for quantitative micro-analysis. Although some information can occasionally be obtained from absorption measurements by using one $\mathrm{X}$-ray wavelength alone, such measurements are more fruitful if two wave-lengths are employed lying on each side of an absorption edge of the element of interest. An apparatus developed for such measurements makes use of an X-ray focal spot about $1 \mu$ in diameter and a crystal monochromator with a proportional counter as detector of the transmitted radiation. It has been used for the determination of calcium in rock sections and in biological materials. Emission spectroscopy, with a somewhat similar apparatus except that the monochromator is now placed out of the primary X-ray beam, has also been used for determination of calcium in these specimens; it seems likely to be more sensitive to small amounts of a given element than the absorption technique, but to be less well fitted to provide accurate information about the distribution of the element in a thin section.

The remaining four papers were concerned with structural investigations. Mr. R. Maitland (King's College, Newcastle upon Tyne) dealt with the crystal structures of chromous and chromic fluorides. The trifluoride structure was determined with little difficulty from powder photographs; it is rhombohedral and of the vanadium trifluoride type, with regular $\mathrm{CrF}_{6}$ octahedra. In chromium difluoride, however, the symmetry is only monoclinic, and the $\mathrm{CrF}_{6}$ octahedra are distorted, giving three different chromium-fluorine bond-lengths ; the structure may be regarded as a distortion of a rutile-type structure.
The differences in bonding between the two fluorides can be completely accounted for by ligand field theory.

Mr. S. Hosoya (University College, Cardiff) discussed the structures of two isomers of thianthrene dioxide, which are of interest as examples of sulphoxides, about which there has been much recent controversy. Only two isomers of thianthrene dioxide have been found ; the $\alpha$-isomer, which has the higher melting point and smaller dipole moment of the two, has been shown by two-dimensional X-ray studies to have the anti-cis form as predicted by Taylor' ${ }^{1}$ and the $\beta$-isomer to have the trans form. Three-dimensional studies of both isomers are in progress to provide detailed information about the sulphur bonding and the folding of the molecules for comparison with other sulphoxides.

Mr. R. Hine (University College, Cardiff) described a determination of the crystal structure of $(+) \mathrm{S}$. methyl-L-cysteine-S-oxide. This substance crystallizes as colourless orthorhombic laths; the space group is $P 2_{1} 2_{2} 2_{1}$, with $a=5 \cdot 2, b=7 \cdot 4, c=16 \cdot 3 \mathrm{~A}$. and the molecular arrangement consists of pairs of sheets parallel to (001) with an elaborate arrangement of short non-bonded distances between certain atoms of the molecules in each sheet. The structure contains an optically active carbon of known configuration, and thus the determination is, in effect, one of the absolute configuration of an asymmetric sulphur atom. The structure of the (-) S-isomer is now being investigated to provide further information about the $\mathrm{S}-\mathrm{O}$ bond.

Mr. H. C. Watson (College of Science and Technology, Manchester) had made use of the optical transform techniques developed in the Physics Department of the College in determining the structure of $\beta$-naphthol from X-ray data. The space group of the crystal is $I a$, and the unit cell has $a=8 \cdot 185, b=5.950, c=36.29$ A., with $\beta=119^{\circ}$ 52 . Projections parallel to the 100 and to the 010 axis were calculated independently; these two projections left a choice between only two passible structures, the projections parallel to 100 and 010 of which were identical but different parallel to 001 . The choice between them was made on the basis of intermolecular distances, an oxygen-oxygen distance of $1.8 \mathrm{~A}$. in one structure being rejected in favour of $2.7 \mathrm{~A}$. in the other, and verified by examining the intensities of a number of general reflexions $h k l$ in an oscillation photograph about the $b$-axis.

R. L. GORDON

1 Taylor, T. W. J., J. Chem. Soc., 625 (1935). 